\title{
Evaluación de los factores de riesgo ergonómico en puestos de trabajo
}

\section{Evaluation of ergonomic risk factors in workstations}

\author{
MARTÍNEZ-SOTO, Ma. Trinidad*†, ESTRADA-FONSECA, María Isabel y GONZÁLEZ-LÓPEZ, \\ Andrés Eduardo
}

Instituto Tecnológico Superior de Irapuato

ID $1^{\text {er }}$ Autor: Ma. Trinidad, Martínez-Soto / ORC ID: 0000-0001-8757-9059, Research ID Thompson: X-3865-2019, CVU CONACYT ID: 691445

ID $1{ }^{\text {er }}$ Coautor: María Isabel, Estrada-Fonseca / ORC ID: 0000-0002-1729-7248

ID 1 ${ }^{\text {er }}$ Coautor: Andrés Eduardo, González-López / ORC ID: 0000-0002-4130-0371

DOI: $10.35429 /$ JTO.2019.10.3.19.23

Recibido 10 de Marzo, 2019, Aceptado, 30 de Junio, 2019

\begin{abstract}
Resumen
La presente investigación está destinada a evaluar el nivel de riesgo de los factores ergonómicos en puestos de trabajo mediante el uso de software y métodos de análisis para adoptar medidas preventivas que mitiguen el riesgo en los trabajadores y cumplir con la normativa vigente aplicable. Para llevar a cabo dicha investigación, este fue apoyado en la metodología DMAIC, iniciando con un diagnóstico en los puestos de trabajo para identificar posibles factores de riesgo presentes y recopilando la información necesaria para simular las actividades a través del Modelado Digital Humano en CATIA® V5 (versión estudiantil) con el método RULA o en el software online CheckList OCRA de Ergonautas ${ }^{1}$. Finalmente, los resultados obtenidos arrojaron la evidencia del nivel de riesgo en cada puesto de trabajo, identificando posibles áreas de oportunidad. Tan pronto las áreas de oportunidad fueron identificadas, estas contribuyeron a plantear las posibles mejoras que dieron pautas para establecer medidas de prevención y rediseño, que pudieran mitigar el riesgo inherente expuesto de los trabajadores en los puestos, permitiendo ajustar ergonómicamente el área y las actividades al trabajador, y a su vez, alineando las prácticas de manufactura a la legislación actual.
\end{abstract}

DMAIC, RULA, OCRA

\begin{abstract}
The present research is intended to evaluate the level of risk of ergonomic factors in workstations through the use of software and analysis methods to adopt preventive measures that mitigate risk in the workers and comply with current applicable regulations. To carry out such research, the project was relied on the DMAIC methodology, starting with a diagnosis at workstations to identify possible present risk factors and collecting the necessary information to simulate the activities through Digital Human Modeling in CATIA® V5 (student version) with the RULA method or in the OCRA CheckList online software of Ergonautas ${ }^{1}$. Finally, the results obtained showed the evidence of the level of risk in each workstation, identifying possible areas of opportunity. As soon as the areas of opportunity were identified, these contributed to set up the possible improvements that gave guidelines to establish prevention and redesign measures, that could mitigate the inherent risk exposed to workers in stations, allowing ergonomically to adjust the area and the activities to the worker and in turn, aligning manufacturing practices to current legislation.
\end{abstract}

DMAIC, RULA, OCRA

Citación: MARTíNEZ-SOTO, Ma. Trinidad, ESTRADA-FONSECA, María Isabel y GONZÁLEZ-LÓPEZ, Andrés Eduardo. Evaluación de los factores de riesgo ergonómico en puestos de trabajo. Revista de Operaciones Tecnológicas. 2019. 3-10: $19-23$

\footnotetext{
* Correspondencia del Autor (Correo electrónico: mamartinez@itesi.edu.mx)

$\dagger$ Investigador contribuyendo como primer autor.
} 


\section{Introducción}

Durante desarrollo de las actividades en la industria, se llevan a cabo labores que conllevan el manejo manual de cargas o tareas con una alta frecuencia de movimientos, es por ello que la existencia de lesiones y/o trastornos musculoesqueléticos dentro de las actividades confiere un problema de salud preocupante y de lo más común en la actualidad. Por tanto, un papel importante que juega la ergonomía en las estaciones de trabajo, es la aplicación de los principios ergonómicos con la finalidad de ajustar ergonómicamente las actividades y el entorno a los trabajadores, y es a través de métodos de análisis postural (RULA-Rapid Upper Limb Assessment) y análisis de repetitividad (OCRA-Occupational Repetitive Actions), los que permiten analizar las actividades presentes en las estaciones para obtener el riesgo al que se exponen los trabajadores en las labores, y del mismo modo, da pautas para el mejoramiento del entorno de trabajo. Así mismo, se da cumplimiento a lo establecido en el artículo 42 del Reglamento Federal del Seguridad e Salud en el Trabajo al contar con los análisis de los Factores de Riesgo Ergonómico expuestos en los trabajadores.

Y puesto que, hoy en día la tecnología mediante la simulación de las estaciones de trabajo, tanto para el RULA a través del Modelado Humano Digital en Catia ${ }^{\circledR}$ V5 (versión estudiantil) y el OCRA en el software online CheckList OCRA en Ergonautas.com nos facilita realizar de una manera más eficiente estudios de ergonomía, así como analizar la información con mayor flexibilidad y rapidez. De modo que usando de referencia una empresa del sector alimenticio para la aplicación de los métodos ya mencionados, se analiza la información de 4 estaciones de trabajo para evidenciar el riesgo expuesto de las actividades a manera de ajustar ergonómicamente la estación al trabajador.

El presente artículo se divide en diferentes sesiones comenzando por la introducción donde se explica el tema general y el objetivo de la investigación. Posteriormente en la descripción del método se detalla la aplicación del método DMAIC, seguido por el apartado de resultados donde se muestran las puntuaciones arrojadas por las simulaciones en los softwares y finalmente las conclusiones obtenidas de la realización de dicha investigación.

\section{Descripción del método}

Utilizando la metodología DMAIC, es por medio del cual en cada una de sus fases se establece el desarrollo del proyecto de investigación.

En la etapa de definir, a través de la observación en el centro de trabajo se identificaron las estaciones que se someterán al análisis, obteniendo cuatro áreas de oportunidad clave donde se observaron focos ergonómicos de atención, ya que se tenía presencia de manejo manual de cargas y/o repetitividad en la tarea, los cuales, se observan en la tabla 1.

\begin{tabular}{|l|l|l|}
$\begin{array}{c}\text { Puesto } \\
\text { de } \\
\text { trabajo }\end{array}$ & \multicolumn{1}{|c|}{ Descripción } & Factor de riesgo \\
\hline P5 & $\begin{array}{l}\text { Pre-doblado manual de } \\
\text { pliegos de cartón, para } \\
\text { alimentación a la } \\
\text { máquina de formado. }\end{array}$ & $\begin{array}{l}\text { Repetitividad - } \\
\text { Levantamiento }\end{array}$ \\
\hline Línea 1 & $\begin{array}{l}\text { Estibado de cajas de } \\
\text { producto terminado en } \\
\text { pallet. }\end{array}$ & Levantamiento \\
\hline Latería & $\begin{array}{l}\text { Estibado de paquetes de } \\
\text { producto terminado en } \\
\text { pallet. }\end{array}$ & $\begin{array}{l}\text { Repetitividad - } \\
\text { Levantamiento }\end{array}$ \\
\hline Fresco & $\begin{array}{l}\text { Alimentación de materia } \\
\text { prima a contenedor de } \\
\text { banda de entrada. }\end{array}$ & Levantamiento \\
\hline
\end{tabular}

Tabla 1 Puestos de trabajo analizados

Del mismo modo, se identificaron las condiciones físicas ambientales que debían contrastarse conforme a las NOM's aplicables, en los diferentes puestos, la iluminación, el ruido y la temperatura, así como la verificación de la aplicación de la NOM-036-1-STPS-2018.

Consecuente a esto, en la etapa de medir, se realizaron las mediciones pertinentes a los factores ambientales, así como se tomaron las dimensiones de la estación de trabajo para su diseño en el software. De igual manera, se tomó evidencia para el filtrado de información, a fin de obtener los datos posturales para el análisis postural, e información de tiempos y movimientos para el estudio de repetitividad. Con los métodos de evaluación ergonómica, se analizó el nivel de riesgo presente en cada uno de los puestos, siendo estos: el RULA para evidenciar el riesgo postural al cual están sometidos los trabajadores en sus labores, y el OCRA para obtener el nivel de riesgo expuesto por la frecuencia y movimientos en las tareas del puesto de trabajo. 
Para realizar de una manera más eficiente los análisis, se apoyó en softwares de simulación, para el riesgo postural se desarrolló a través del Modelado Humano Digital en el software Catia ${ }^{\circledR}$ V5 (versión estudiantil) para el diseño del entorno de trabajo de cada estación de trabajo (véase en el apartado anexos, ilustración 1) y para la simulación de las diferentes posturas (véase en el apartado anexos, ilustración 2). En cuanto al análisis de repetitividad, se sometió mediante un software online CheckList OCRA, disponible en Ergonautas.com, donde se insertaron los datos requeridos para el análisis y se procedió a simulación para arrojar los resultados de la información, con lo cual se obtuvo el nivel de riesgo (véase en el apartado anexos, ilustración 3).

En la parte de mejorar, se plantearon consideraciones de rediseño del área de trabajo, así como cambios en el método de trabajo para disminuir los niveles de riesgo en cada una, a manera de adecuar el puesto al trabajador, regulando al mismo tiempo las actividades conforme a la legislación actual.

Como última fase de controlar, se realiza un seguimiento posterior a las mejoras para llevar un registro de lo mejorado, y de igual forma, tener en cuenta posibles riesgos futuros que puedan surgir, ya que las modificaciones en las restricciones pasadas pueden presenciarse nuevos acontecimientos por resultar impredecibles e imperceptibles en el pasado.

\section{Resultados}

Los resultados de las evaluaciones RULA y OCRA (véase la tabla 2), así como de la verificación de los factores de riesgo conforme a las NOM's aplicables (véase la tabla 3), se filtraron en un ergograma a manera de organizar y visualizar mejor la información.

\begin{tabular}{|c|c|c|c|c|}
\hline \multicolumn{5}{|c|}{ Resúmen de las evaluaciones ergonómicas } \\
\hline $\begin{array}{l}\text { Puesto } \\
\text { de } \\
\text { trabajo }\end{array}$ & Posturas & $\begin{array}{l}\text { RUL } \\
\text { A }\end{array}$ & $\begin{array}{l}\text { OCR } \\
\text { A }\end{array}$ & \begin{tabular}{|l} 
NO \\
M- \\
036- \\
$1-$ \\
STP \\
S- \\
2018 \\
\end{tabular} \\
\hline \multirow{3}{*}{ P5 } & Postura 1: Carga & 3 & & \\
\hline & Postura 2:Descarga & 5 & & \\
\hline & $\begin{array}{l}\text { Postura 3: Repetitividad (Pre- } \\
\text { doblado manual) }\end{array}$ & & 20.9 & \\
\hline \multirow{3}{*}{$\begin{array}{l}\text { Línea } \\
1\end{array}$} & Postura 4: Carga & 7 & & \multirow{3}{*}{8} \\
\hline & Postura 5: Estiba nivel 1 & 7 & & \\
\hline & Postura 6: Estiba nivel 6 & 6 & & \\
\hline \multirow{4}{*}{$\begin{array}{l}\text { Laterí } \\
\text { a }\end{array}$} & Postura 7: Carga & 5 & & \multirow{4}{*}{8} \\
\hline & Postura 8: Estiba nivel 1 & 7 & & \\
\hline & Postura 9: Estiba nivel 20 & 4 & & \\
\hline & $\begin{array}{l}\text { Postura 10: Repetitividad (carga- } \\
\text { descarga }\end{array}$ & & 18.9 & \\
\hline \multirow{3}{*}{$\begin{array}{l}\text { Fresc } \\
\text { o }\end{array}$} & Postura 11: Carga nivel 1 & 6 & & \multirow{3}{*}{7} \\
\hline & Postura 12: Carga nivel 7 & 4 & & \\
\hline & Postura 13: Descarga & 4 & & \\
\hline
\end{tabular}

Tabla 2 Ergograma "evaluaciones ergonómicas"

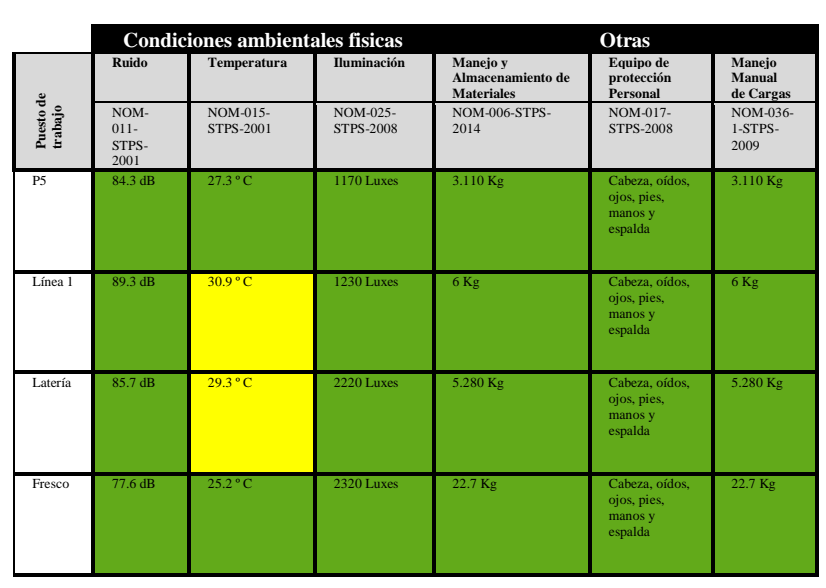

Tabla 3 Ergograma "NOM's"

\section{Conclusiones}

Al realizar el análisis de los diferentes puestos de trabajo con los métodos de evaluación correspondientes, se evidencio el alto nivel de riesgo ergonómico que implica la realización de sus respectivas actividades, todo esto derivado de tres factores importantes: el peso de la carga y posturas forzadas obtenido de la simulación RULA, así como de la evaluación del riesgo mediante la NOM-036-1-STPS-2018 y la repetitividad de la operación observado a través del método OCRA.

Además, como se pudo identificar en cada uno de los estudios analizados, las posturas inadecuadas son variables e influyen negativamente en el trabajador, si bien el adoptar las posturas de trabajo adecuadas y un buen manejo de cargas manuales no elimina el riesgo ergonómico de la actividad, si lo disminuye considerablemente.

MARTÍNEZ-SOTO, Ma. Trinidad, ESTRADA-FONSECA, María Isabel y GONZÁLEZ-LÓPEZ, Andrés Eduardo. Evaluación de los factores de riesgo ergonómico en puestos de trabajo. Revista de Operaciones Tecnológicas. 2019 
Con el propósito de disminuir al mínimo el riesgo ergonómico producido por las actividades, es recomendable capacitar y adiestrar al personal involucrado en cuanto al correcto manejo manual de cargas, aunque es importante señalar que aun haciendo uso de estas prácticas el riesgo sigue inherente a la actividad, por tanto, se deben atacar otros factores relevantes como lo es el peso, ya que es el principal influyente en nivel riesgo de exposición en el manejo de cargas, además de concientizarlos sobre el impacto que tienen las buenas posturas de trabajo.

De una manera más profunda, es conveniente rediseñar el entorno y/o método de trabajo introduciendo sistemas de estibado automatizados como brazos robóticos, así como aplicar la rotación del personal y mejoras en la tarea haciendo uso de la economía de movimientos.

\section{Agradecimientos}

Un atento agradecimiento al Instituto Tecnológico Superior de Irapuato por el financiamiento proporcionado para el desarrollo del proyecto en el marco del CICA2019.

\section{Referencias}

1. Diego-Mas, Jose Antonio. OCRA Check-List. Ergonautas, Universidad Politécnica de Valencia, 2015. Disponible online: https://www.ergonautas.upv.es/metodos/OCRA /ocra_online.php

Aldaco Quijas, A., \& Pérez Salas, O. (2017). Rediseño de estaciones de trabajo para el cumplimiento de las NOM STPS, Conagra Brands S. de R.L. de C.V. (Tesis Pregrado). Instituto Tecnológico Superior de Irapuato. México.

Baroja Payán Erica, J. R. (2015). Aplicación de la técnica RULA en el área de empaquetado mediante tecnología Kinect. Revista Iberoamericana para la Investigación y el Desarrollo Educativo.

Mcatamney, L., \& Corlett, E. N. (1993). RULA: A survey method for the investigation of workrelated. Applied Ergonomics.
Mohamad, D. D. (2013). RULA Analysis of Work-Related Disorder among Packaging Industry. Switzerland: Trans Tech Publications. Akyol, S. D., \& Baykasoğlu, A. (2019). ErgoALWABP: a multiple-rule based constructive randomized search algorithm for solving assembly line worker assignment and balancing problem under ergonomic risk factors. Journal of Intelligent Manufacturing, 30(1), 291302.

Asadi, H., Yu, D., \& Mott, J. H. (2019). Risk factors for musculoskeletal injuries in airline maintenance, repair \& overhaul. International Journal of Industrial Ergonomics, 70, 107-115.

Munguía Vega, N. E., Flores Borboa, V. S., Zepeda Quintana, D. S., \& Velazquez Contreras, L. E. (2019). Assessing the effectiveness of integrating ergonomics and sustainability: a case study of a Mexican maquiladora. International Journal of Occupational Safety and Ergonomics, 25(4), 587-596.

Sanjog, J., Patel, T., \& Karmakar, S. (2019). Occupational ergonomics research and applied contextual design implementation for an industrial shop-floor workstation. International Journal of Industrial Ergonomics, 72, 188-198.

Zetterberg, C., Heiden, M., Lindberg, P., Nylén, P., \& Hemphälä, H. (2019). Reliability of a new risk assessment method for visual ergonomics. International Journal of Industrial Ergonomics, 72, 71-79.

\section{Anexos}

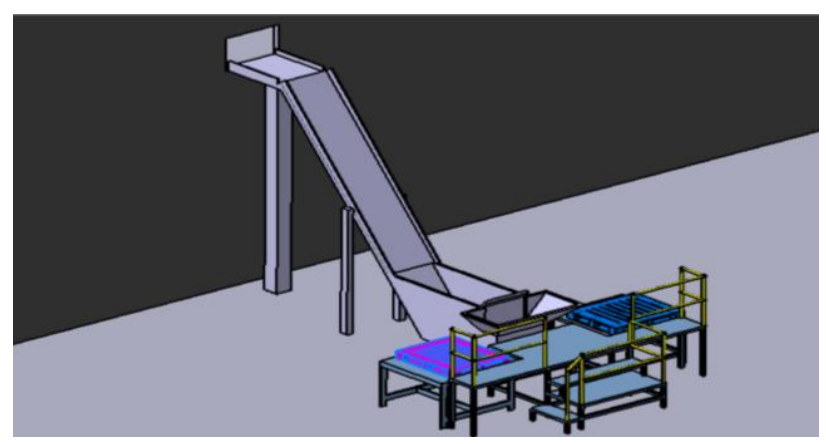

Figura 1 Representación gráfica del área de trabajo "Fresco" 


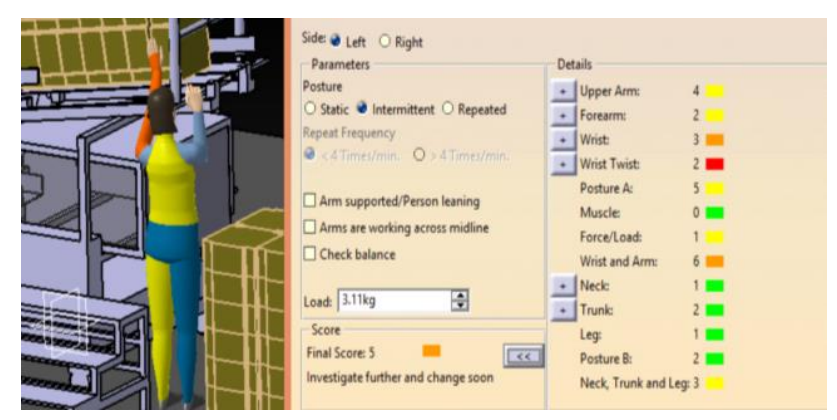

Figura 2 Representación gráfica de la simulación RULA "P5"

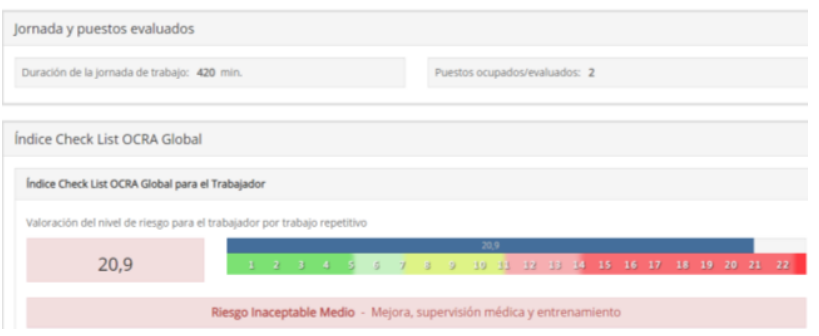

Figura 3 Resultados de la simulación OCRA "Latería" 